\title{
EL USO DE MAPAS CONCEPTUALES COMO HERRAMIENTA EDUCATIVA EN EL ÁMBITO DE LOS NÚMEROS RACIONALES
}

\author{
CONTRERAS, L.C. \\ Departamento de Didáctica de Las Ciencias (Experimentales, Sociales y Matemáticas). \\ Universidad de Huelva. Facultad de Ciencias de la Educación. Avenida de las Fuerzas Armadas, s/n. \\ 21071 Huelva.
}

\section{SUMMARY}

This article is a synthesis of a research carried out as a final project included in a Doctoral Program. The mentioned research consisted of a case study whose goal was to highlight the methodological advantages of conceptual maps in initial diagnosis, instructional design and assessment, as described in the corresponding literature. The identification of rational numbers as those with a finite or periodical decimal representation has been chosen as the specific topic. The individuals were studying the third degree of Teachers' Training.

«Si tuviera que reducir toda la psicología de la educación a un sólo principio, diría esto: el factor sencillo más importante que influencia el aprendizaje es lo que ya sabe el que aprende. Averíguielo y enséñele en concordancia con ello.»

Ausubel, 1968.

\section{INTRODUCCIÓN}

Esta preocupación de Ausubel tenía antecedentes en la obra de Bartlett (1932). También Kelly (1955) enfatizó el papel del conocimiento previo en el nuevo aprendizaje, pero sin marcar la importancia de los conceptos específicos ni de las tramas proposicionales.

El tomar como punto de partida la estructura conceptual de los alumnos (Giordan, 1978) tiene una especial relevancia en el modelo de enseñanza que se puede deducir de esta visión constructivista y que se interesa en la forma en que los nuevos conocimientos pueden integrarse en dicha estructura.

Los problemas de esa integración plantean alguna complejidad. Las ideas previas de los aiumnos ofrecen una gran resistencia al cambio, por un lado, porque resultan, adecuadas para interpretar determinados aspectos de su campo de experiencias $\mathrm{y}$, por otro (aunque en relación con lo anterior), por el margen de racionalidad que poseen dado el grado de coherencia que suelen presentar (Clement, 1982; Driver, 1986). Por ello no son fácilmente alterables por un proceso de enseñanza tradicional (que no los tenga en consideración) y no son de fácil acceso, dado que ni siquiera los propios alumnos no las hacen explícitas para ellos mismos, en bastantes casos. Como contrapartida a esta dificultad, podríamos decir que el abanico de concepciones tiene una cierta limitación, en el sentido de que se detectan ideas similares en estudiantes de diferentes contextos sociales y cronológicos.

En los últimos años se han utilizado diversos procedimientos para estudiar, tanto los conocimientos del alumno antes de comenzar una instrucción determinada, como 
los instrumentos más adecuados para llevar a cabo (partiendo de los datos anteriores) dicho proceso instructivo y la manera de ilustrar los cambios cognoscitivos producidos.

De entre los sistemas utilizados para poner de manifiesto los conocimientos previos, podemos citar los socráticos (Champagne, Gunstone y Klopfer, 1983); la discusion entre los estudiantes (Gilbert y Pope, 1982); la resolución de problemas en pequeños grupos (Whimbey y Lochhead, 1980).

También se han utilizado los tests de «lápiz y papel», aunque existe un consenso sobre la ineficacia de este sistema, que tan sólo parece poner de relieve en torno al $10 \%$ de los conocimientos previos de los alumnos (Novak, 1988b). Otro instrumento mucho más fiable ha sido la entrevista clínica piagetiana con determinadas modificaciones (Pines et al., 1978), cuyos principales obstáculos estriban en la subjetividad y la transcripción c interpretación (además de que no puede utilizarse como elemento de evaluación rutinaria durante la clase).

En un intento de obtener la citada información por otros conductos, numerosos autores han usado la estrategia del mapa conceptual (Cardemone, 1975; Novak, 1988a; Novak y Gowin, 1984; Hoz, 1987; Hoz, Kozminsky y Bowman, 1987; Álvarez y Risco, 1987). Novak y sus colaboradores comenzaron a utilizar varias formas de "mapas conceptuales», bien para representar las tramas conceptuales derivadas de estructuras proposicionales procedentes de una entrevista clínica, bien claborados directamente por los alumnos para expresar las mismas (Novak, 1988b). Paulatinamente se fue comprobando que el diseño de mapas conceptuales se manifestaba útil como instrumento para explicitar los esquemas conceptuales de los alumnos en un determinado momento y en un área de conocimiento concreta. Kstos resultados dieron pie a investigar de qué manera podrían también convertirse en un elemento coadyuvante en la planificación de la instruccion y del currículo, y como instrumento válido para la evaluación (Moreira y Novak, 1988).

\section{UN ESTUDYO DE CASOS EN EL ÁMBITO DF LA IDENTIFICACION DE LOS NUMMEOS RACIONAIES}

\section{Objetivos de la investigación}

De entre todos los aspectos señalados más arriba, nuestro estudio tenía como objetivo realizar una aproximación a las siguientes cuestiones:

1. Verificar la eficacia de los mapas conceptuales para reflejar los conceptos, sus significados y sus relaciones.

Fs decir, verificar si los mapas conceptuales elaborados por los sujetos, en relación con un determinado núcleo de conceptos, reflejarán los significados que cada uno otorga a los conceptos intervinientes y las relaciones (en cuanto a la calidad y cantidad) entre los mismos.
2. Analizar la posibilidad de establecer una hipótesis de progresión ${ }^{1}$ para la construcción de ese núcleo de conceptos a partir de la información que procede de los mapas. Olo que es to mismo: una vez puesta de manifiesto la cantidad y calidad de las relaciones, será posible establecer una hipótesis de progresión conceptual (que emane de un mapa conceptual de referencia) que, de ser validada inicialmente por la información obtenicia a través de los mapas de los alumnos en la fase de exploración (para cuya interpretación serviría de referencia), se convertirá en una guía de las rutas de aprendizaje a seguir.

3. Analizar la eficacia de los mapas conceptuales para medir la eventual evolución (en cuanto a aprendizaje conceptual de los sujetos) derivada de un determinado proceso de instrucción.

Se trata de estudiar si los mapas conceptuales, elabora* dos por los sujetos durante un proceso de instruccion diseñado à amparo de la hipótesis de progresión, darán información sobre la evolución de cada uno en relación con la citada hipótesis y los efectos de la instrucción recibida.

\section{Aspectos y consideraciones previas}

Para tratar de dar respuesta a estas cuestiones era necesario, no obstante, detenerse en algunos aspectos previos.

\section{Determinar el núcleo conceptual a estudiar}

Elegimos la identificación de los números racionales como los únicos números reales con representación decimal finita o periódica. Nos pareció que, en el desarrollo escolar de la estructura numérica, era una etapa que parece tener un interés capital. Además de la problemática propia de dicha caracterización, pensábamos que poseŕa un interés particular debido fundamentalmente a dos razones:

- Por un lado, lo podíamos calificar como econcepto bisagra” (en cuanto a su distribución temporal a lo largo de la enseñanza obligatoria), dado que, en la estructura de $E G B$, se iniciaba su tratamiento en $8^{\circ}$, pero no se concluía su estudio en este curso, sino que los aspectos de inconmensurabilidad de algunos segmentos ${ }^{2}$ eran pospuestos para los primeros cursos de BUP, quedando, por tanto, la caracterización completa a caballo entre dos niveles educativos, dos filosofías de trabajo escolar distintas y dos concepciones de aprendizaje no necesariamente comunicadas. En este sentido cabría decir que no existen razones aparentes para dicho tratamiento y que incluso pođría dar luz al hecho de que la caracterización de los racionales sigue siendo un problema para la mayoría de los estudiantes que culminan los estudios preuniversitarios.

- Pero, desde otro punto de vista, el análisis histórico de este concepto (Boyer,1968; Collette,1973) fundamen- 
talmente en cuanto al estudio de los inconmensurables, parece determinarlo como uno de los más complejos de los albores de la matemática, que de hecho prácticamen. te demolía las bases de la fe pitagórica en los números enteros (Boyer, 1968, p. 106) ${ }^{3}$.

Nuestra experiencia docente en los primeros cursos de formación del profesorado nos muestra que lo que pare. cen haber aprendido los alumnos respecto de este concepto después de la instrucción en el bachillerato es poco más que:

- identificación del conjunto de números naturales, aunque no se sabe muy bien por qué unas veces se incluye el cero y otras no;

- identificación del conjunto de números enteros $\mathrm{y}$ asunción de que $\mathrm{N} \subset \mathrm{Z}$ ( $\mathrm{e}$ incluso que $\mathrm{N}=\mathrm{Z}^{+}$). A veces suelen ignorar la necesidad de la construcción de $\mathrm{Z}$;

- reconocimiento de $\mathrm{Q}$ como el conjunto de números fraccionarios. Aquí, el hecho de que $Q$ sea un conjunto de representantes puede plantear problemas para identificar la relación $Z \subset Q$. Además no justifican la necesidad de construir $Q$;

- memorísticamente reconocen la relación $\mathrm{N} \subset \mathrm{Z} \subset \mathrm{Q} \subset$ $R$, a veces se dan cuenta de que en realidad hay más rúmeros como $\sqrt{ } 2, \pi$ o el número $e$, que recuerdan que no eran racionales, pero no saben muy bien por qué.

A nuestro entender, son los dos puntos anteriores los cruciales en la caracterización de Q; de hecho, podemos decir que una correcta integración del concepto en el esquema aritmético de número exige:

- reconocer a $\mathrm{Q}$ como un conjunto de representantes;

- comprender que todos los números racionales son los únicos que poseen una representación decimal finita o periódica.

En el contex to escolar es frecuente observar que el paso de decimal a fracción a través de la fracción generatriz se limita al uso (de forma memorística) de las reglas de conversión, sin haber justificado el procedimiento que nos lleva a ellas. Hemos comprobado que los alumnos de primer curso de ciencias de la Escuela de Formación del Profesorado recuerdan la existencia de dichas reglas aunque son incapaces de eponerlas en pie» $y$, por supuesto, de justificarlas.

Por último, una correcta caracterización de los racionales, en la línea de lo expuesto anteriormente, debe traducirse en aplicaciones concretas, entre las que queremos resaltar la capacidad de representación de cualquier número en la recta real y la resolución de problemas aritmético-geométricos donde el número racional esté presente.

Pensamos que el problema radica, más que en la dificultad intrínseca de estos conceptos, en la falta de conexiones necesarias en las estructuras mentales correspon- dientes, conexiones que no suelen aparecer cuando se utiliza un tipo de instrucción como el que habitualmente se emplea para el desarrollo de estas nociones en el contexto escolar, y que los mapas conceptuales podrían detectar y soslayar.

\section{Elegir los individuos objeto de estudio}

Optamos por elegir los sujetos de entre aquéllos sobre los que se tuviera un fácil acceso (en el sentido de disponibilidad) y que además, por su nivel de estudios, pudieran otorgar significados ricos al campo conceptual elegido.

Por otro lado, aunque estábamos dispuestos a entrenar a los sujetos en el uso de mapas conceptuales en otros ámbitos distintos al elegido, la fase de entrenamiento 4 pudo obviarse, puesto que los mapas conceptuales habían sido tratados como parte del temario y en coordinación con las asignaturas Didáctica de las Ciencias Experimentales y Didáctica de las Ciencias Sociales (Contreras y Estepa, 1994) en un tercer curso de Formación del Profesorado de Primaria. Este hecho, junto a la decision de optar por un estudio de casos dado el carácter cualitativo de la investigación y la inexistencia de intención de extrapolar sus conclusiones a otros contextos, nos llevó a solicitar un grupo de voluntarios, de entre los citados alumnos, para lievar a cabo el estudio.

Por otro lado, este hecho permitía a este grupo de alumnos (cuatro) ${ }^{5}$ aplicar y constatar algunas de las posibilidades, por ellos estudiadas, en el campo instruccional y profundizar en un núcleo conceptual del que, a priori, suponíamos que tenđría escaso significađo para ellos por la problemática descrita anteriormente.

\section{Diseño de la investigación}

La primera fase consistió en la elaboración de un mapa conceptual de referencia sobre el núcleo elegido (Cuadro I). Éste nos permitiría establecer el grado de aproximación de cada individuo a nuestro modelo conceptual en cada parte del proceso. Además serviría de guía para la elaboración de la hipótesis de progresión (Cuadro 2).

En una segunda fase realizamos una exploración inicial sobre los conocimientos de los sujetos en relación con el núcleo elegido, usando mapas conceptuales. De cada sujeto obtuvimos varios borradores antes de la «versión definitivas.

Consideramos necesario situar a cada sujeto con relación a su nivel de evolución respecto de la hipótesis de progresión ${ }^{6}$. Esto constituyó la tercera fase.

En la cuarta fase diseñamos y pusimos en práctica una instrucción coherente, tanto con los datos obtenidos como con la filosofía del proyecto. Ésta fue elaborada a la luz de láa propia hipótesis de progresión. Los cuadros 4 y 5 pretenden sintetizar el proceso de enseñanzaaprendizaje investigativo en el que participaron exclusivamente los sujetos del estudio. Una descripción detallá 
da puede encontrarse en el informe completo de este estudio (Contreras, 1992).

Durante el proceso elegimos dos momentos para la obtención de datos, en su segunda mitad y al final. Se usaron, para ello, nuevos mapas conceptuales elaborados por los sujetos sobre el núcleo en cuestion. Ésta es la quinta y útima fase.

\section{Metodología dela investigación. Instrumentode recogida de datos}

Para obtener información sobre la estructura conceptual de estos alumnos en el desarrollo del concepto de número racional y poder analizar en qué medida los mapas conceptuales pueden ser válidos como instrumentos detectores, en este núcleo conceptual concreto, la primera sesión consistió en una propuesta de elaboración de un mapa conceptual personal con objeto de analizar los siguientes elementos:

- Semánticos, en el sentido de conocer el significado que los alumnos otorgan a una lista de conceptos.
- De contexto, en cuanto a las relaciones que los alumnos establecen entre los citados conceptos.

La sesión se celebró en una mesa de trabajo para seis plazas. Desde el centro de Ja misma, se podía ver con facilidad el desarrollo del trabajo de cada uno. A cada sujeto se le dio una lista no jerarquizada compuesta por los conceptos o términos conceptuales que aparecen en el mapa (Cuadro 1), así como por los ejemplos intercalados entre aquéllos de forma intencionadamente desordenada. Las relaciones buscadas son las que se han mostrado en el cuadro 1 .

Con objeto de obtener información sobre el grado de significado de los conceptos, se ofrecían una serie de ejemplos (los que aparecen en el cuadro 1 junto a sus términos conceptuales) concretos de los mismos, que los alumnos tendrían que utilizar en la elaboración de su mapa conceptual.

Con el propósito de aportar el mayor número posible de datos en relación con el proceso de razonamiento que seguían en su tarea, se pidió a los alumnos que formulasen todo tipo de cuestiones aclaratorias, con la única

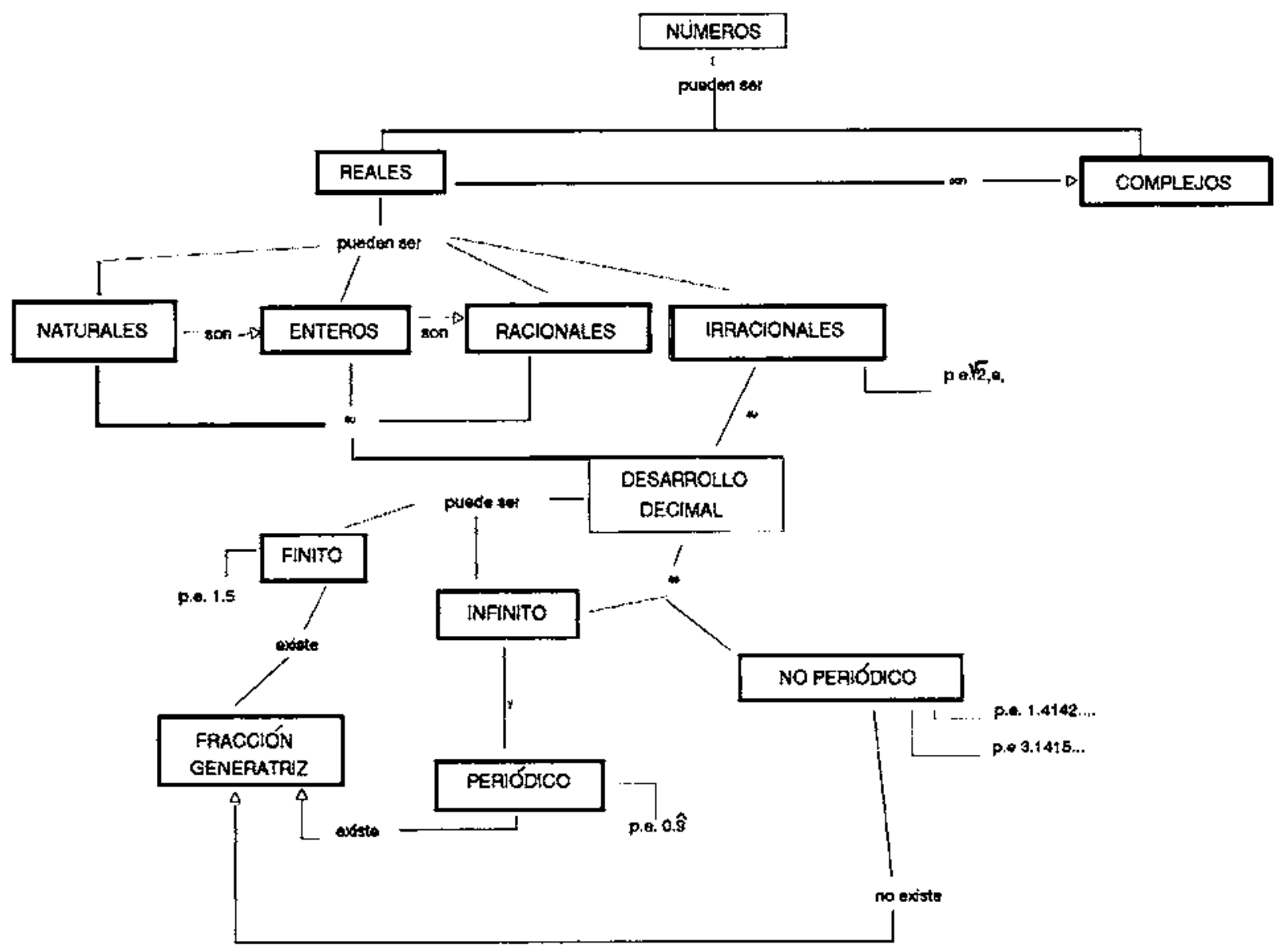


Cuadro 2

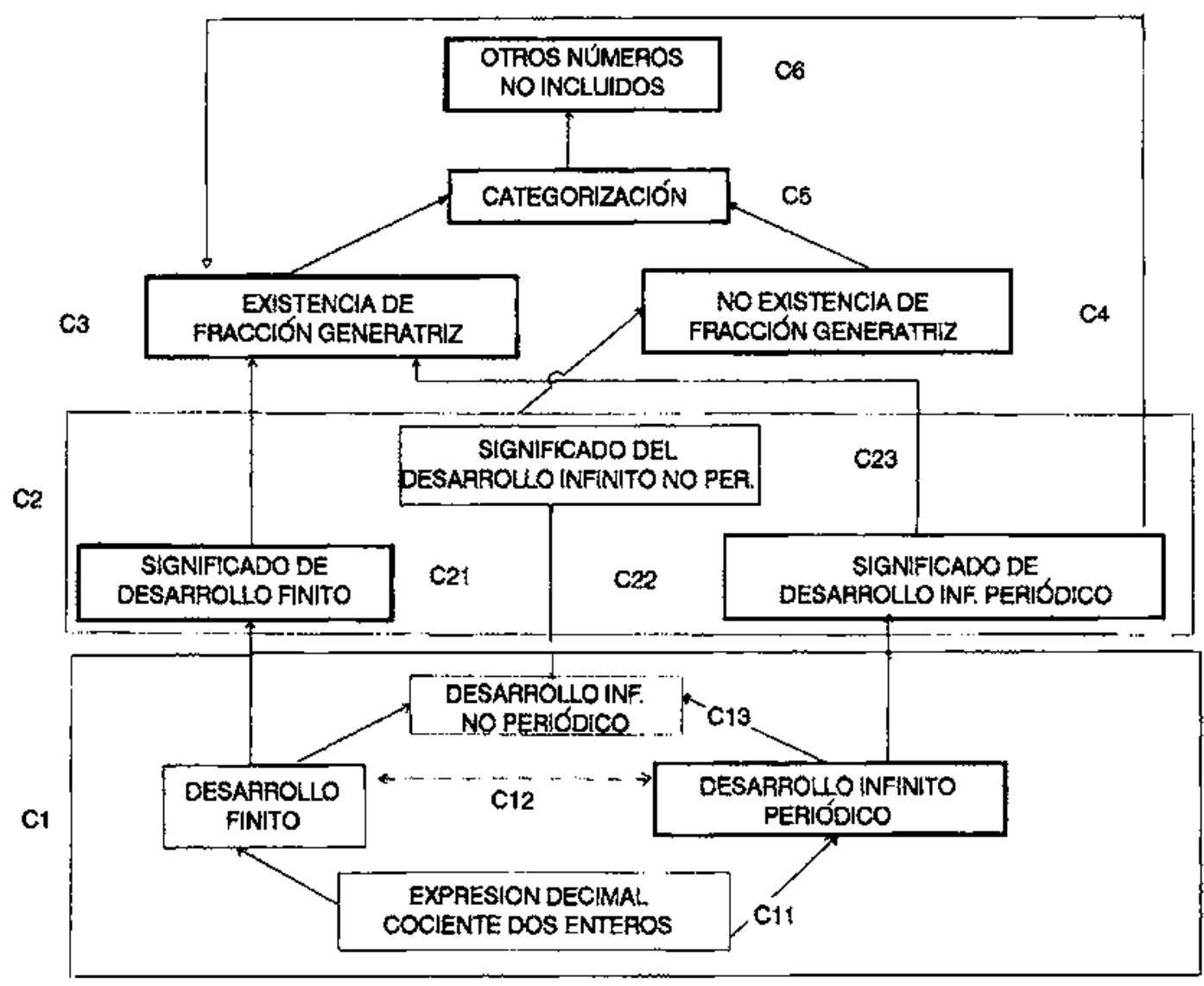

condición de que las registraran por escrito. De alguna manera, intentábamos recoger aspectos locales del proceso de razonamiento (a modo de las secuencias o fotogramas de una película), que los mapas podrían no plasmar.

En una segunda sesión, y una vez realizado un primer análisis de los mapas elaborados por los alumnos?, se mantuvo una sesión de intercambio, al comienzo de la cual se facilito a cada alumno el mapa conceptual de referencia. El objetivo era el de ayudar a explicitar, aún más, tanto los significados como las posibles relaciones. Para ello se les pedía que expresaran, en un turno individual, aquellos aspectos que observaban en el mapa de referencia en contradicción o desacuerdo, bien con su punto de vista, bien con lo que habían expresado en sus correspondientes mapas conceptuales.

Toda la información era recogida en un cuadro de control (Cuadro 3) elaborado al amparo de la hipótesis de progresion que emana del mapa conceptual de referencia.
Cuadro 3

\begin{tabular}{|l|l|l|l|l|l|l|}
\hline MAPADATOS & $\mathrm{Cl}$ & $\mathrm{C} 2$ & $\mathrm{C} 3$ & $\mathrm{C} 4$ & $\mathrm{C5}$ & $\mathrm{C6}$ \\
\hline CORRECTO & & & & & & \\
\hline INCORRECTO & & & & & & \\
\hline AUSENTE & & & & & & \\
\hline
\end{tabular}

El análisis de los mapas en la fase de diagnóstico nos permitió hacer una validación inicial de la hipótesis de progresión así como diseñar una instrucción que queda sintetizada en el cuadro 4

Como puede observarse, los aspectos que aparecen en recuadros hacen alusión a elementos de la hipótesis de progresión (Cuadro 2) y debajo de éstos figura una nota aclaratoria. El lateral derecho expresa el número de sesiones previstas para cada uno de los recuadros corres- 
pondientes. El esquema siguiente (Cuadro 5) desarrolla con un poco más de profundidad la fase de búsqueda de ejemplos de desarrollos decimales (segundo nivel del gráfico 4). Se corresponde con una lista de actividades cuya caracterización, desde el punto de vista organizativo, se expone en las dos columnas laterales.

Cuadro 4

Esquema del proceso de instrucción.

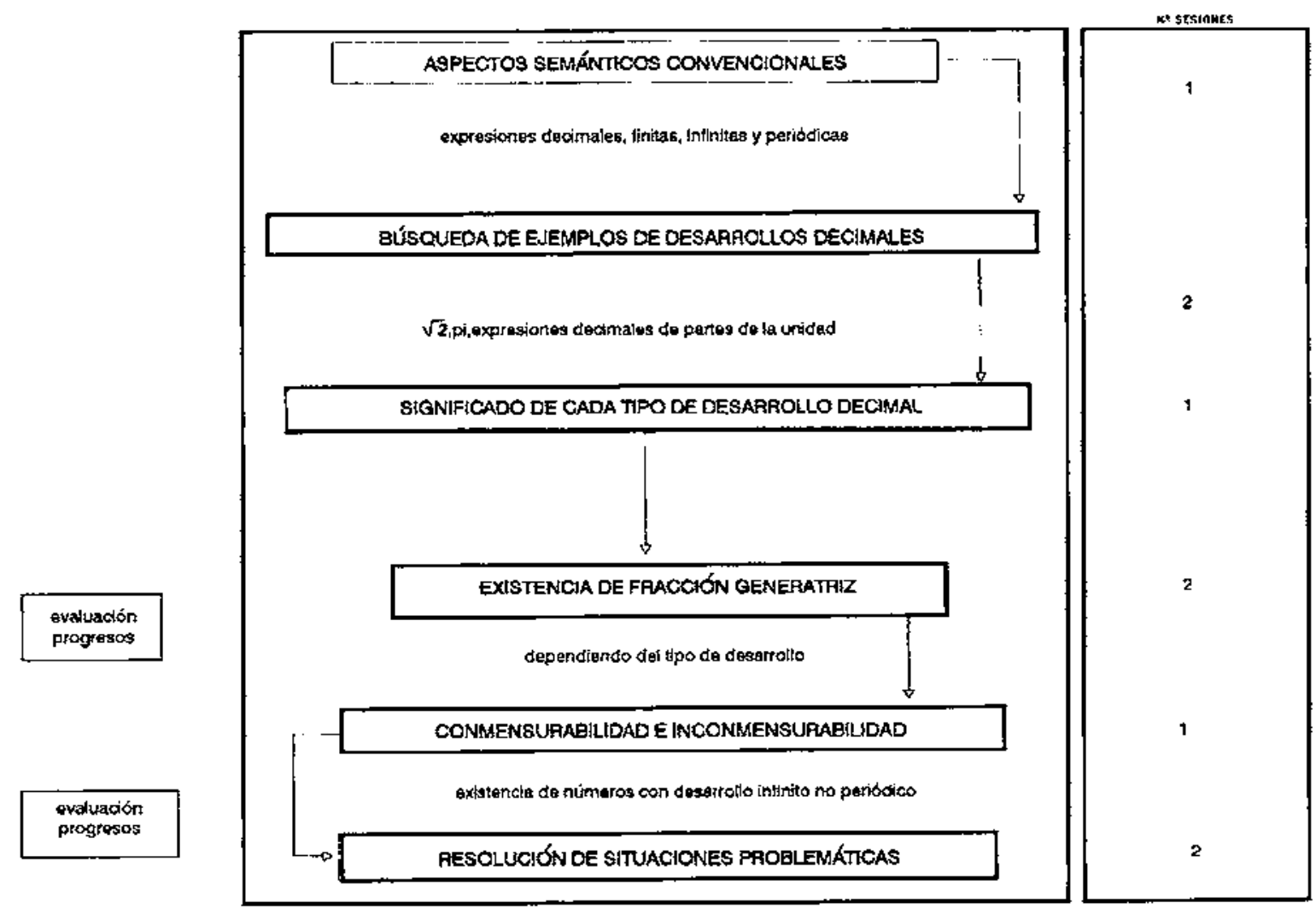

Cuadro 5

Esquema de las sesiones de búsqueda de ejemplos.

\begin{tabular}{|c|}
\hline TIPODE \\
\hline ACTVIOAD \\
\hline individuri \\
indilvidual \\
individuat \\
grupo
\end{tabular}

BUSQUEDA DE EJEMPLOS DE DESARROLLOS DECIMALES

\begin{tabular}{|c|}
\hline $\begin{array}{l}\text { CÁ́lCULO MANUSAL DE RÁ́ OE DOS } \\
\text { con tantos decimales como "sea posible" }\end{array}$ \\
\hline 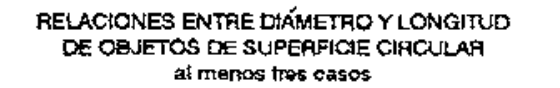 \\
\hline $\begin{array}{c}\text { EXPFESIONES DECIMALES DE } \\
1 / 1,1 / 2,1 / 3,1 / 4,1 / 5,1 / 6,1 / 7,1 / 8,1 / 9,1 / 10 \text { y } 39 / 44\end{array}$ \\
\hline $\begin{array}{l}\text { SIMILITUOES Y CARACTERISTCAS OIFEAENCIALES } \\
\text { DE LOS DESARAOLLOS ENCONIAADOS }\end{array}$ \\
\hline
\end{tabular}

REALIZACIÓN

\section{previo} encango

provlo encargo 
Cuadro 6

Mapa conceptual de Gabriela.

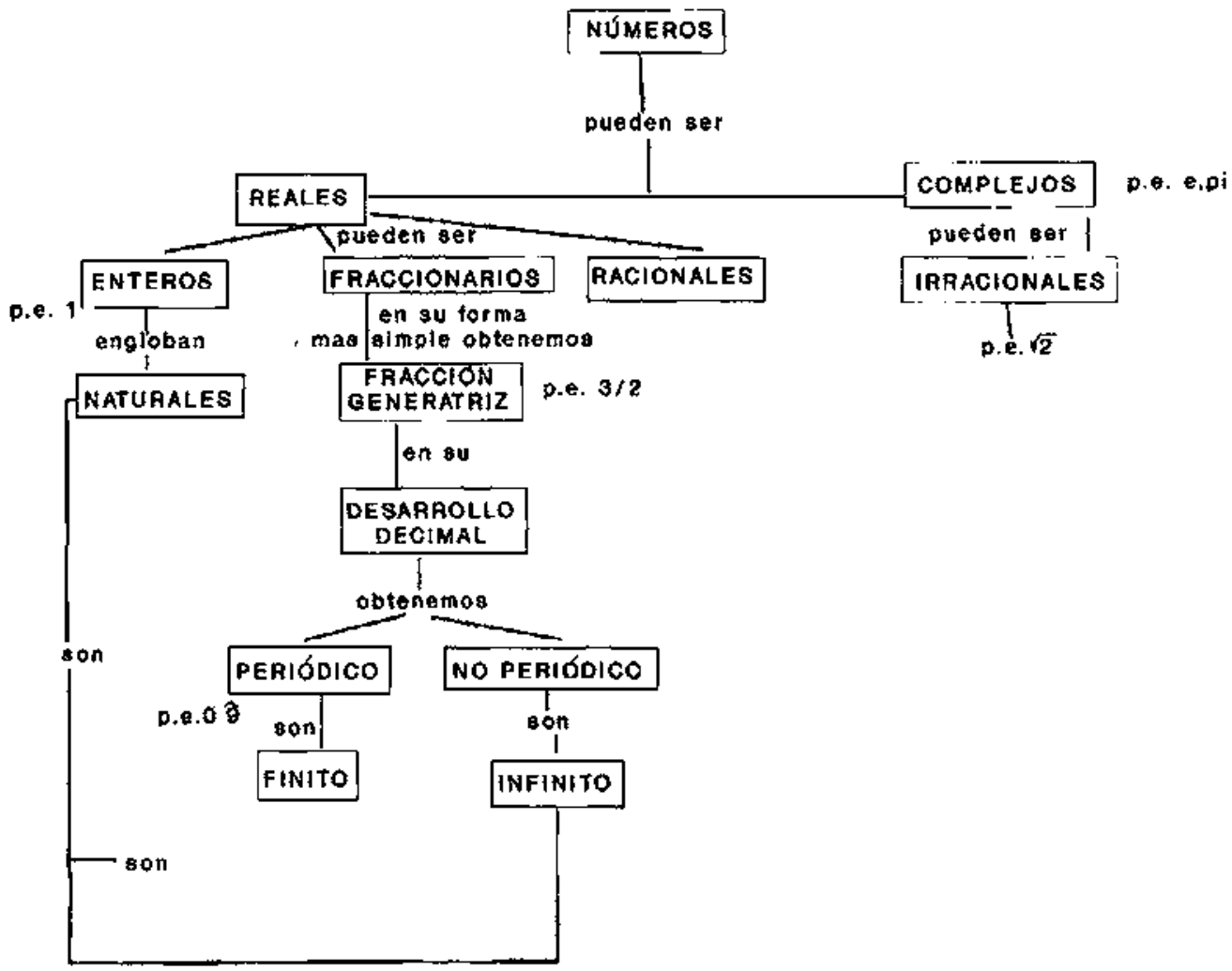

Para reflejar el proceso descrito con anterioridad hemos elegido uno de los casos. No es fácil tomar una decisión cuando todos los individuos ofrecen información relevante, pero este caso refleja una evolución más palpable; es decir, existe un fuerte contraste entre las informaciones obtenidas en cada uno de los momentos de recogida de datos. De todas formas, nos parece que hay algo subjetivo en la elección; no podemos negarlo aunque cueste reconocerlo; un padre siempre tiene preferencias.

\section{Un caso particular: Gabriela}

En la fase de diagnóstico, Gabriela ejecutaba un mapa conceptual cuya transcripción es la siguiente:

\section{(OMFNTARIOS DE GABRIELA EN LA FASE DE DIAGNÓSTICO}

* Los números racionales (no los fraccionarios) son aquéllos que poseen raíz cuadrada «con solución».

" Los números irracionales no poseen raiz cuadrada «exacta».

: $1.4142 \ldots$ es periódico mixto de períndo 4.

* $3.1415 \ldots$ parece $\pi$

* ¿Pueden existir dos números distintos en cuyos desarrol los decimales coincidar sólo las cifras 3,1415 ?

* 0.999... es 1 por aproximación.

\section{DISCREPANCIAS}

* Ś́lo los fraccionarios tienen desarrollo decimal.

*No veo la relación entre la existencia de fracción generatriz y el carácter no períbdico de un número

*Los únicos números con desarrollo infinito son tos no períódicos.

*Los números fraccionarios y los racionales son bistintos.

* Los números complejos pueden ser irracionales.

* Los númcros reales no son un caso de los complejos.

El reflejo de los datos en un cuadro de control es el siguiente:

Cuadro 7

\begin{tabular}{|c|c|c|c|c|c|c|}
\hline MAPA/DATOS & $\mathrm{Cl}$ & $\mathrm{C} 2$ & $\mathrm{C} 3$ & $\mathrm{C} 4$ & $\mathrm{CS}$ & $\mathrm{C} 6$ \\
\hline CORRECTO & & & & & & \\
\hline INCORRECTO & & & & & & \\
\hline AUSENTE & & & & & & \\
\hline
\end{tabular}

El único concepto que se identifica como correcto en su mapa es el correspondiente a la expresión decimal del 
cociente de dos enteros (C11). El mapa conceptual pone de relieve, además, las siguientes incorrecciones:

- concepto de desarrollo decimal finito o periódico (C12);

- concepto de desarrollo decimal infinito no periódico (C13);

- significados de desarrollos decimales fínito e infinito periódico (C21, C22);

Notemos que el concepto de desarrollo finito e infinito se asocia al concepto de número natural, posiblemente estableciendo el carácter finito de cada elemento, e infinito asociado al cardinal de $\mathrm{N}$.

Los comentarios evidencian otros errores conceptuales:

- los dos primeros ponen de manifiesto un error de categorización de los números racionales e irracionales;

- el tercero evidencia un error en el concepto de número períbdico (C12);
- Ia tercera discrepancia respecto al mapa de referencia revela error en el concepto de desarrollo infinito no periódico (C13);

- en ese mismo sentido, Ia segunda discrepancia manifiesta errores de significado (C23) e identificacion $(\mathrm{C} 3, \mathrm{C} 4)$.

Como ya comentamos en el apartado 2.3, el mapa conceptual que se ofrece (Cuadro 6) es la última versión. Los borradores son tambiên muy ilustrativos:

Ya hemos comentado que se evidencia, en los comentarios y cuestiones anexas a los mapas conceptuales, una categorización semántica incorrecta, en el sentido de que, a pesar de que conoce la mayor parte de los nombres de los conceptos enunciados, no tiene bien delimitadas las clases subyacentes a dichos conceptos. Gabriela parece otorgar un contenido semántico a periódico, pero afirma que $1,4142 \ldots$ lo es de período 4 .

Incluso en el reconocimiento del nombre del concepto hay un problema en cuanto a la relacion racional/fraccionario. La no identificacion podría argumentarse en razón de aspectos etimológicos, sociológicos o epistemológi-

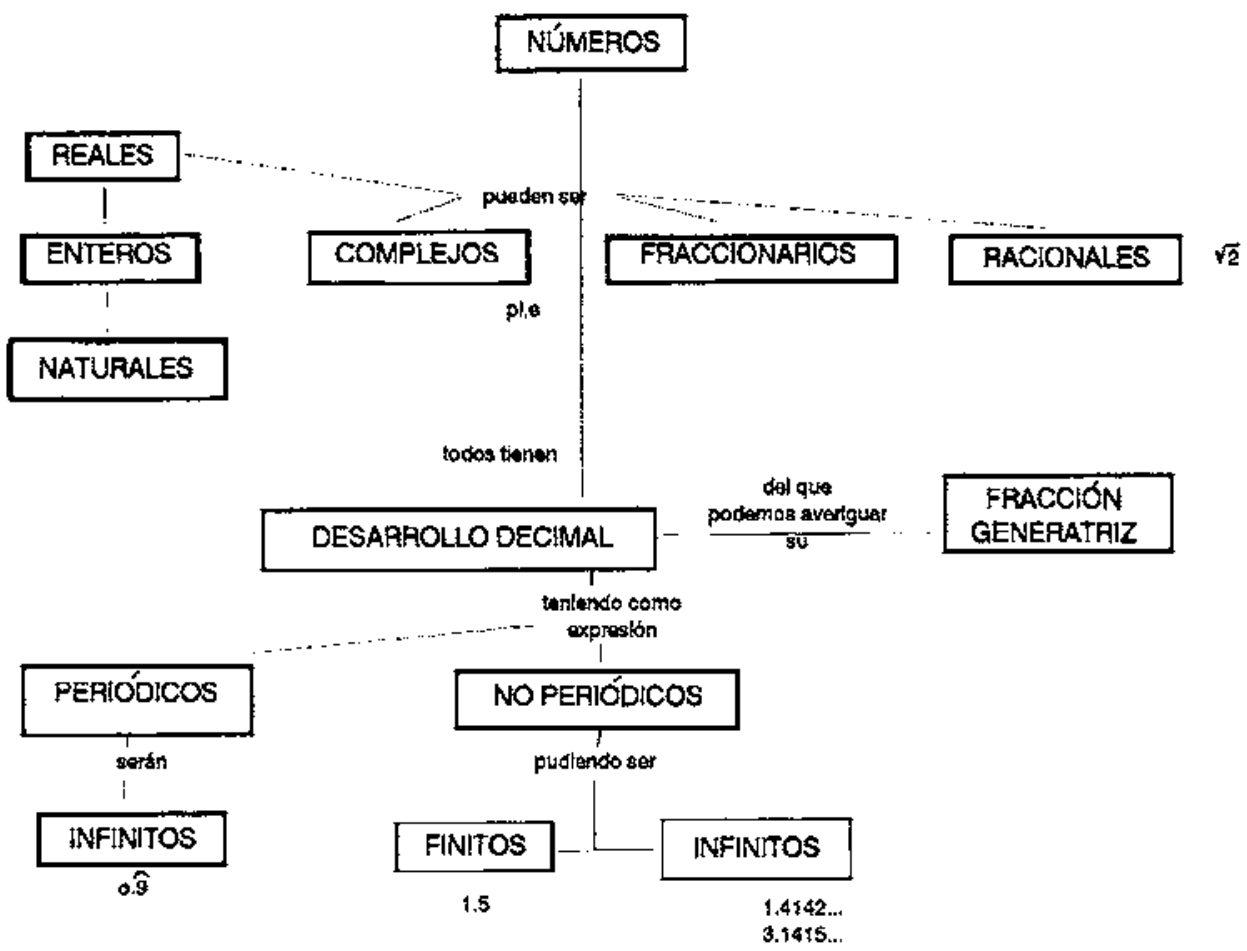


Cuadro 9

Scgunda vcrsión deI mapa de Gabriela en la fase de diagnóstico. El cambio responde a una cuestión de estrategia para no repetir conceptos y establecer mejores relaciones.

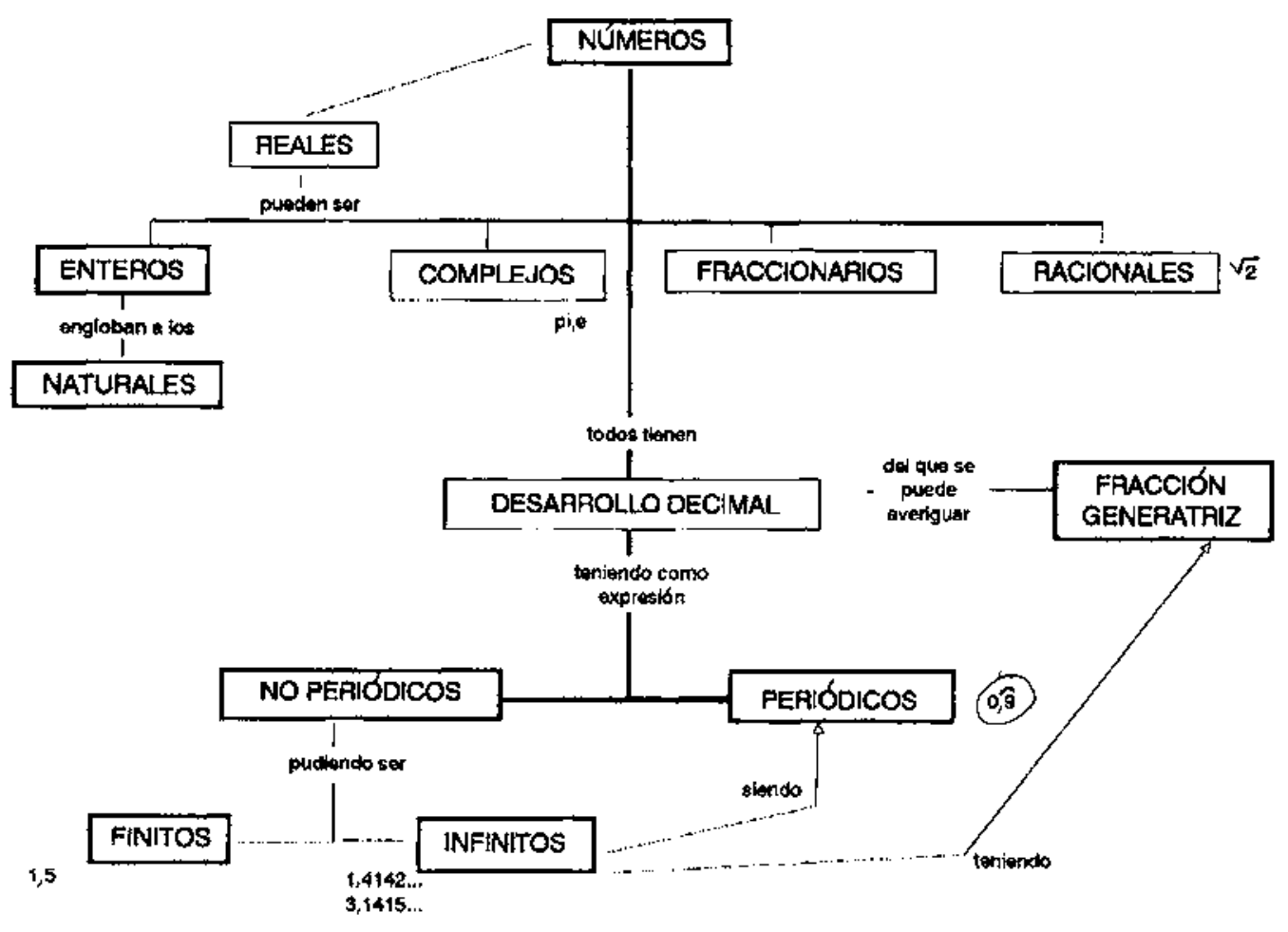

cos (Giménez, 1991); pero sea cual fuere la razón, es claro que distorsiona las relaciones con otros conceptos del mapa.

Resulta curioso el escaso significado otorgado a la expresión fracción generatriz. Gabriela me afirmaba poco después su identificación con fracción irreducible.

De todas maneras, sólo los fraccionarios (en un sentido estricto y como su propio nombre indica) parecen poder representarse como fracciones y como decimales. Este dato, combinado con la ausencia de relación entre naturales y enteros y entre éstos y los racionales (en los mapas), sugiere que, en la construccion de dichos conjuntos, Gabriela no ha reconocido ei hecho de que cada nuevo conjunto nace, a partir del anterior, para poder resolver una gama de situaciones aritméticas irresolubles en el conjunto de partida.

Los números e y $\pi$ no parecen ser del mismo tipo que $\sqrt{2}$. De hecho, saberros que no lo son (trascendentes los primeros y algebraico el segundo), pero ella desconoce la verdadera razón. Estimo que se apoya en el hecho de que los primeros no evocan ninguna operación habitual en ellos. De los comentarios de Gabriela se podría deducir que atribuye a los trascendentes un carácter de «inventados» (por necesidad científica), mientras que a $\sqrt{2}$ se le otorga una existencia «cierta».

Hay una idea de aproximación en aiguno de los comentarios. Así, Gabriela dice «3, $1415 \ldots$ parece $\pi » 0 \ll 0,999 \ldots$ es 1 por aproximación».

El mapa que sigue se corresponde con el elaborado por Gabriela en la última sesión (Cuadro 10). En él podemos observar los cambios producidos con respecto al elaborado en la fase de diagnóstico.

Lo más significativo del cuadro 10 es que se completa el nivel $\mathrm{C} 1$ de la hipótesis de progresión, con los subniveles $\mathrm{C} 12$ y $\mathrm{C} 13$ (posibilidades de expresión decimal de un número real), así como el significado de desarrollo finito (C21). Se desvincula el concepto de fracción generatriz de número fraccionario (C3). Además vemos cómo se han completado las subcategorías C22 y C23, admitiendo $\sqrt{2}$ como número con desarrollo no periódico; se 
Cuadro 10

Mapa final de Gabriela.

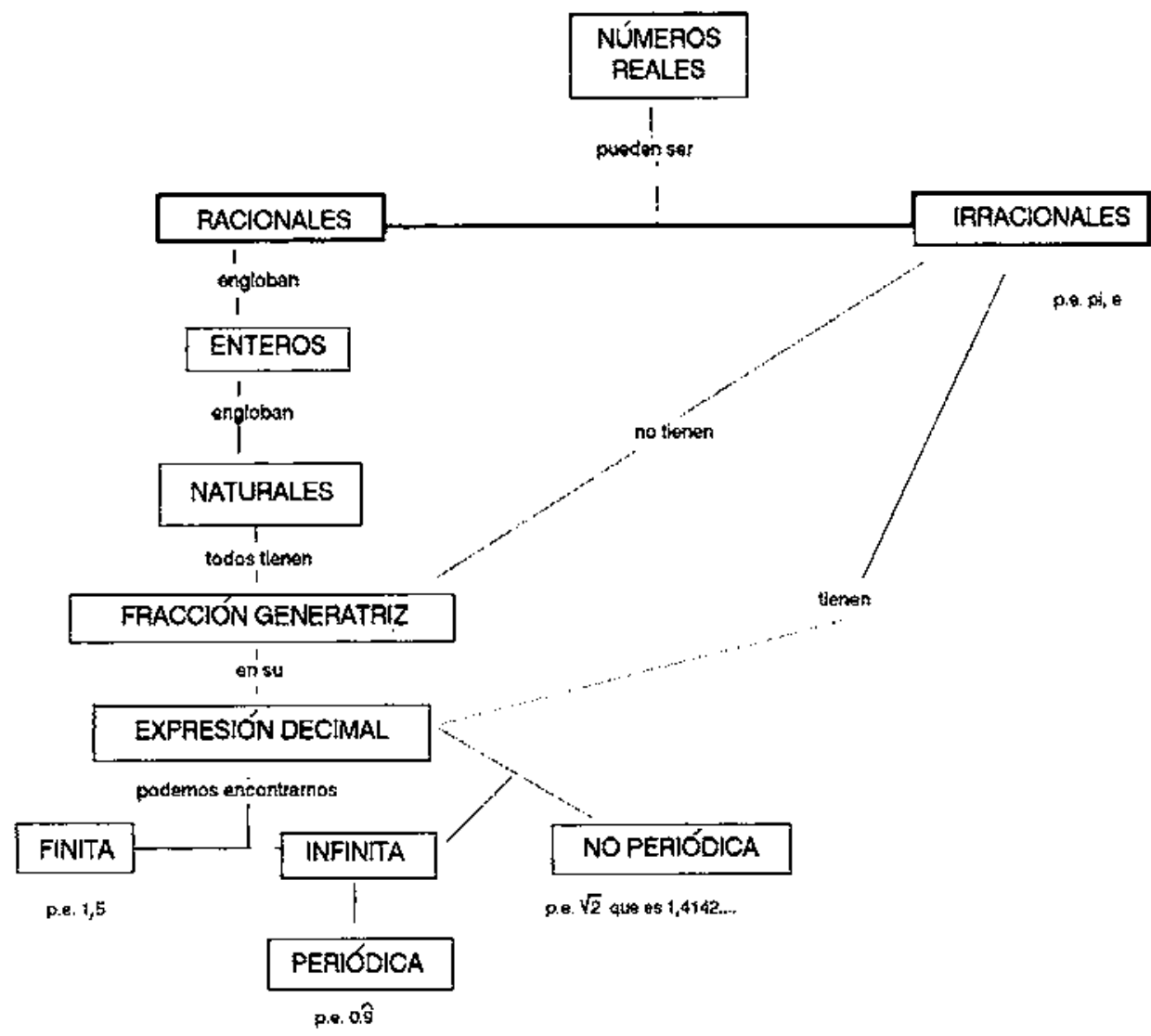

otorga significado al concepto de fracción generatriz (C3) y se establece que sólo los racionales poseen tal fracción (C3 y C4). Ese aspecto es el que le permite una jerarquía correcta (C5).

\section{REFLEXIONES FINALES}

A modo de síntesis, invitamos a reflexionar sobre los siguientes aspectos:

a) Los mapas conceptuales, como instrumento de diagnóstico, parecen ser más potentes si se utilizan conjuntamente con otros medios, como, por ejemplo, la entrevista, aunque nuestra opción intermedia ha sido el diálogo con los alumnos (registro manual), en base a sus mapas, sus comentarios durante la realización de éstos y el contraste efectuado por los alumnos con el mapa de referencia. La información obtenida a través de estos otros medios (y que hemos expuesto al principio de este epígrafe) es suficientemente rica como para justificar esta afirmación. Pero además, a través de los mapas, es difícil observar otros aspectos no conceptuales, como los procedimientos. b) Como instrumento de diseño instruccional, los mapas conceptuales nos han permitido trazar una ruta de aprendizaje no convencional, como muestra la validación de la hipótesis de progresión conceptual elaborada a partir de ellos. No obstante, la instrucción por este proceso ađquiere un prominente carácter conceptual y aquellas tareas de aprendizaje donde el alumno adoptó un papel de investigador fueron marcadamente dirigidas. Ello hace necesario disponer de elementos adicionales a los mapas a la hora de diseñar el proceso de enseñanzaaprendizaje.

c) Los mapas conceptuales elaborados por los alumnos durante el proceso de instrucción han ofrecido una información fiable de los efectos de ésta sobre aquéllos, tanto en los aspectos positivos (progresión) como negativos, en el sentido de que pueden reflejar intervenciones negativas del profesor durante el proceso.

d) El proceso ha permitido a los alumnos mejorar la utilización de mapas para reflejar su pensamiento. Paulatinamente, sus mapas han ido siendo un mejor reflejo de la realidad, en el sentido de que conceptos que inicialmente incluían sin significado han terminado por omitirse. 


\section{NOTAS}

I El término hipótesis de progresión es utilizado habitualmente para poner de relieve una secuencia teórica de los aprendizajes previstos en un núcleo concreto.

2 Adoptamos la terminología griega, entendiendo el número como magnitud y conceptualizando los irracionales como la medida de aquellos segmentos que no pueden conmensurarse con los conocidos hasta ese momento.

3 Como anécdota, a un pitagórico relevante, Hipaso de Crotona, lo expuisaron de la «hermandad», al parecer por revelar el descubrimiento de los inconmensurables, por sus presuntas consecuencias devastadoras para la filosofía pitagórica (Boyer, 1968, p. 165).

* Hemos optado por seguir el proceso indicado por Novak y Gowin (1984), del que poseemos datos experimentales, y que en cursos de formación inicial del profesorado de primaria se ha desartollado en torno a cuatro sesiones de noventa minutos.

\section{REFERENCIAS BIBLIOGRÁFICAS}

ÁLVAREZ, M. y RISCO, V. (1987). Concept Maps and Vee Diagrams: A Visual Representation of Children's Thinking. Paper presented at AERA meetings. Washington, D.C.

AUSUBEL, D. (1968). Educational Psychology: A Cognitive View. Nueva York: Holt, Rinehart, and Winston. (Traducción, 1976, Psicología Educativa: un punto de vista cognitivo. México: Trillas).

BARTLETT, C. (1932). Remembering. Cambridge, Mass.: Cambridge University Press.

BOYER, C.B. (1968). A History of Mathematics. Nueva York: John Wiley and Sons. (Traducción, 1986, Historia de la matemática, Madrid: Alianza Editorial).

CARIDEMONE, P. (1975). Concept Mapping: A Technique of Analyzing a Discipline and its Use in the Curriculum and Instruction in a Portion of a College Level Mathematics Skills Cottrse. Tesis de Master inédita. Ithaca, Nueva York: Cornell University, Department of Education.

CLEMENT, J. (1982). Student alternative conceptions in mechanics: a coherent system of reconceptions, en Helem, H. y Novak, J. (cds.). Proceedings of the International Seminar "Misconceptions in Science and Mathematics". Ithaca, Nueva York: Cornell University.

COLLETTE, J.P. (1973). Histoire des mathématiques. Montréal: Editions du Renouveau Pédagogique. (Traducción, 1985, Historia de las matemáticas, Madrid: Siglo XXI).

CONTRERAS, L.C. (1992), El uso de mapas conceptuales como in strumento de diagnóstico inicial, diseño instruccional y evaluación. Un estudio de casos en la representación decimal de los números reales. Memoria de Tercer Ciclo. Departamento de Didáctica de las Ciencias. Universidad de Sevilla.
5 Por cuestiones de espacio, aunque nos referiremos a la muestra en plural, sólo mostraremos los datos relativos a uno de sus elementos, Gabriela. La elección está justificada por la riqueza de aspectos que ofrece

6 Como puede verse, la hipótesis de progresión que obtenemos a partir del mapa conceptual de referencia, es utilizada en diferentes momentos de la investigación. En primer lugar, para comprobar el nivel conceptual en el diagnóstico inicial; en segundo lugar, para elaborar una propuesta instruccional; $y$, finalmente, en dos momentos distintos, para evaluar la evolución de los alumnos. De alguna manera, por tanto, la citada hipotesis constituye el modelo de conocimiento matemático escolat deseable en el tópico elegido.

7 Este primer análisis es superficial; es una primera toma de contacto con los conceptos y relaciones, así como tubicación de ejemplos, en los mapas de los sujetos y del grado de homogeneidad de los mismos.
CONTRERAS, L.C. y ESTEPA, J. (1994). Aportación interdisciplinar para la delimitación de un cuerpo de conocimiento común de las didácticas especiales a través de Ios mapas conceptuales, en Montero, L. y Vez, J.M. (eds.). Las didácticas específicas en la formación del profesorado, II, Vol. II. Santiago: Tórculo Edicións.

CHAMPAGNE, A., GUNSTONE, R., KLOPFER, L. (1983). Effecting changes in cognitive strtictures amongst physics students. Paper presented at the Symposium on Stability and the American Association, Montreal.

DRIVER, R. (1986). Psicología cognoscitiva y esquemas conceptuales de los alumnos. Enseñanza de las Ciencias, $4(1), \mathrm{pp} .3-15$

GILBERT, J. y POPE, M. (1982). School children discussing energy. Report of Institute of Educational Development, University of Surrey.

GIMÉNEZ,J. (1991). Innovación metodológica de la didáctica especial del número racional positivo. Tesis doctoral inédita. Universitat Autònoma de Barcelona.

GIORDAN, A. (1978). Une pédagogie pour les sciences expérimentales. París: Editions di Centurion. ('Iraducción, 1982, La enseñanza de las ciencias. Madrid: Siglo XXI).

HOZ, R. (1987). Dimension of Teacher's Knowledge Structure and Their Identification by Concept Mapping. Paper presented at AERA Meetings, Washington, D.C.

HOZ, R., KOZMINSKY, E. y BOWMAN, D. (1987), Evaluating Learning Through Concept Mapping: A Study of Student's Cognitive Structure in Earth Science Course. Unpublished paper, Ben Gurion University, Beer-Sheva, Israel. 
KEIJ,Y, G.A. (1955). The Psychology of Personal Constructs, $1 \& 2$. Nueva York: W.W. Norton and Co.

MOREIRA, M.A. y NOVAK, J.D. (1988). Investigación en Enseñanza de las Ciencias en la Universidad de Cornell: esquemas téricos, cuestiones centrales y abordes metodológicos. Enseñanza de las Ciencias, 6(1), pp. 3-18.

NOVAK, J.D. (1988 a). Constructivismo humano: un consenso cmergente. Enseñanza de las Ciencias, 6(3), pp. 213-223.

NOVAK, I.D. (1988 b). Fi constructivismo humano: hacia la unidad en la elaboración de significados psicológicos y epistemológicos, en Porlán, R., García, J.E.,Cañal,P. (Comps.) Constructivismo y enseñanza de las ciencias. Sevilla: Díada.

NOVAK, J.D. y GOWIN, D.B. (1984). Learning how to Learn. Nueva York: Cambridge University Press. (Traducción, 1988, Aprendiendo a aprender. Barcelona: Martínez Roca).

PINES, A. et al. (1978). The Clinical Interview: A Method for Evaluating Cognitive Structures. Research Report 6. Ithaca, Nueva York: Corneli University, Department of Education.

WHIMBEY, A. y LOCHHEAD, J. (1980). Problem Solving and Comprehension. Filadelfia: Franklin Institute Press.

[Artículo recibido en marzo de 1996 y aceptado en noviembre de 1996.] 